\title{
LA LUCILIA SERICATA EN THÉRAPEUTIQUE
}

\author{
Par André MAURICE
}

Les myiases, ou invasion des plaies ou des organes par les larves de mouches, comptent parmi les affections les plus anciennement connues; elles comptent aussi parmi les plus redoutables et le fait d'utiliser les larves de mouches dans un but thérapeutique semble à première vue un acte bien audacieux; nous verrons pourtant, d'après les observations qui suivent que ce mode de traitement, dans certaines plaies, donne les résultats les plus satisfaisants.

Nous avons exposé précédemment (1) l'historique de la question, la technique à suivre dans l'application des larves de Lucilia, l'action de ces larves, leur utilisation soit à l'état vivant, soit au moyen des sucs qui en ont été extraits; nous rapporterons seulement, dans cet article, un certain nombre d'observations, qui nous ont permis de formuler quelques conclusions pratiques.

\section{Observations}

Obs. I. Service du prof. Lenormant. - R. C..., âgé de dix-neuf ans, entre à l'hôpital Cochin le 26 mars 1933, pour ostéomyélite chronique du fémur gauche. Il a déjà été opéré en province, le 19 janvier 1933, pour ostéomyélite aiguë de l'extrémité inférieure du fémur. Lorsqu'il est entré dans le service, il n'avait ni fièvre, ni accidents aigus, mais une ostéite fistuleuse ayant largement envahi l'épiphyse fémorale. Il a été pratiqué chez lui, le $1^{\text {er }}$ avril, un évidement avec ablation de séquestres et un curetage de la cavité osseuse. Une autre intervention analogue est pratiquée le 21 juin; la mise à plat est complète, les dimensions de la plaie considérables.

Huit jours après cette opération, c'est-à-dire le 29 juin, on fait une première application de larves de Lucilia sericata; celle-ci est suivie de quatre autres le 6 juillet, le 12 juillet, le 18 juillet, le 24 juillet. Dès la première, s'est produite une modification complète des sécrétions de la plaie qui sont devenues séro-sanguinolentes, de purulentes

(1) Maurice (A.). - La Lucilia sericata en thérapeutique ; larves vivantes ; sucs extraits de larves. Résultats cliniques. Paris, A. Legrand, éd., 1934.

Annales de Parasitologie, T. XIV, $\mathrm{N}^{\circ} 1$ - - $1^{\text {er }}$ janvier 1936, p. 35-47. 
qu'elles étaient auparavant. Des bourgeons vivaces se sont développés avec une extrême rapidité et ont réduit progressivement les dimensions de la cavité, si bien que, lors de la dernière application, quatre semaines après le début du traitement, celle-ci était presque complètement comblée.

Le malade quitte le service le 12 août, ne gardant plus qu'une fistulette insignifiante, qui s'est fermée au début de novembre. Actuellement, depuis un an, la cicatrisation est complète.

OBs. II. Service du prof. Mathiev. - M. V..., âgé de 19 ans. Entre à l'hôpital Saint-Louis, le 8 décembre 1930, pour une ostéomyélite de l'extrémité inférieure du tibia droit. Atteinte marquée de l'état général. Allure septicémique de l'affection. Température à $40^{\circ}$. Le 10 , puis le 17 décembre, incision d'abcès sous-périostés. Le 22 décembre 1930, trépanation totale de la diaphyse, évidement complet, pansement à plat et plâtre. Chute thermique mais propagation à l'articulation tibiotarsienne. Le 2 janvier 1931, diaphysectomie de tout le tibia droit. Astragalectomie. Suppuration pendant les mois de janvier, février, mars et avril. Le $1^{\text {er }}$ mai, il subsiste sur la face interne du tibia une surface de 10 centimètres de long non encore épidermisée et deux petites fistules, l'une en dehors, l'autre en dedans sous chaque malléole. Quitte l'Hôpital Saint-Louis en juillet 1931. Les fistules persistent toujours.

En mars 1932, devant la persistance des fistules, le malade entre à l'Hôpital Cochin dans le service du professeur Mathiev. Le 7 avril 1932, grattage au niveau du tibia. Persistance des fistules. Le 14 mai 1933, grattage au niveau du tibia. La suppuration continue toujours. Le $1^{\text {er }}$ juillet 1933, troisième grattage au niveau du tibia. Mise à plat complète de toute la région.

Le 7 juillet 1933 : Première application de larves de Lucilia sericata. On laisse les larves quatre jours et on fait une nouvelle application le cinquième jour. On fait ainsi régulièrement six applications entre le 7 juillet et le 7 août. La première application a déterminé une réaction thermique, la deuxième un simple clocher, les autres sont à peine marquées sur la courbe de température. Les applications sont très bien supportées; le bourgeonnement est rapide et le malade part en convalescence à Vincennes; la fermeture de la plaie est presque complète.

Le malade est revenu au mois de décembre 1933 : la cicatrisation est parfaite. Une radiographie a été pratiquée le 17 janvier 1934. Nous avons eu des nouvelles du malade au mois de juin 1934; la guérison est complète.

Obs. III. Service du prof. Ombrédanne. - P. P..., âgé de 12 ans, entre à l'hôpital des Enfants-Malades pour une fracture ouverte de la jambe droite, survenue le 23 juin 1933. Opéré le 23 juin, 18 heures après l'accident; le 18 juillet, réduction sanglante d'un chevauchement important. 
Plâtre de Delbet. Sort de l'hôpital le 3 octobre ; la plaie suppure toujours. Le 7 novembre, entre de nouveau à l'hôpital : opéré le 7 novembre. Excision de la plaie fistuleuse; curetage du foyer de fracture, on enlève quatre séquestres.

Le 10 novembre, première application de larves. On fait ainsi successivement six applications de larves, qu'on laisse chaque fois pendant quatre jours; la dernière application est faite le 12 décembre; le 15 décembre la jambe présente une légère lymphangite; on enlève les larves. A partir du 3 janvier 1934, on fait sur la plaie tous les trois jours une application de nitrate d'argent. Le 22 janvier la plaie est cicatrisée.

Obs. IV. Service du prof. Ombrédanne. - E. S..., âgée de 14 ans, entre à l'hôpital des Enfants-Malades, au mois d'août 1933, pour une ostéomyélite subaiguë de l'extrémité inférieure du fémur droit; le début de l'affection remonte à huit mois. La radiographie montre de nombreux séquestres. A cette époque, première intervention et ablation des séquestres. Le 7 novembre 1933, deuxième intervention : excision de la cicatrice antéro-interne. Curetage du fémur; on ne trouve pas de séquestres. Débridement de la fistule située à la face interne du genou. On a ainsi deux plaies distinctes: l'une antérieure et supérieure de 17 centimètres de long et de 8 centimètres de large; l'autre interne et inférieure de $7 \mathrm{~cm}$., 5 de long et 3 centimètres de large.

Première application de larves le 10 novembre. L'enfant subit ainsi sept applications; mais la plaie inférieure se comble trop rapidement, les lèvres bourgeonnent et se rapprochent, empêchant les larves d'accéder jusqu'au fond de la plaie; la fistule subsiste. Une nouvelle mise à plat très large est faite en janvier 1934, avec ouverture du trajet fistuleux; la surface osseuse antéro-interne du fémur est visible sur une grande paume de main. On fait alors huit applications successives de larves; la plaie se comble rapidement; la guérison semble totale à la fin du mois de février 1934. Radiographie le 27 avril 1934. Radiographic le 21 juillet 1934 .

Obs. V. Service du prof. Ombrédanne. - S. C..., àgée de 13 ans 1/2, entre à l'Hôpital des Enfants-Malades, dans le service du prof. OMBrÉDANne, pour une ostéomyélite du cubitus droit. Début brusque, le 26 août 1933, avec violente douleur dans l'avant-bras droit, température à $41^{\circ}$. Frissons. Entrée à l'Hôpital de Romorantin le 30 août 1933. Le 2 septembre, on pratique deux incisions à la face postérieure de l'avant-bras avec grattage de l'os. Le 16 novembre, la malade quitte l'Hôpital de Romorantin et entre à l'Hôpital des Enfants-Malades. A cette date : aucune douleur. Très bon état général. Pas de température. Suppuration continue. Le 18 novembre, on fait quatre radiographies qui montrent : l'intégrité de l'humérus ; d'énormes lésions du cubitus.

Le 24 novembre, intervention chirurgicale par le $\mathrm{D}^{\mathrm{r}}$ Zagdoun : résection de toute la diaphyse cubitale. Le 28 novembre, radiographie. 
A cette date, large plaie losangique occupant toute la face postéricure de l'avant-bras et s'étendant de l'articulation du coude à l'articulation du poignet sur une longueur de 26 centimètres et une largeur, à la face médiane de l'avant-bras, de 10 centimètres.

Le 29 novembre, première application de larves de Lucilia sericata. L'enfant reçoit ainsi 12 applications de larves en trois mois à raison d'une application chaque semaine. Des photographies en séries ont été faites au cours du traitement (Pl. I). Restitution complète de l'os : sur la mince lamelle cubitale, constitution d'un os nouveau, solide, opaque aux rayons X. Fin avril 1934, la plaie est entièrement cicatrisée, le bras a repris la plupart de ses mouvements après mobilisation prudente et progressive. La malade quitte l'Hôpital le 4 mai 1934.

Obs. VI. Servive du prof. Duval. - R. G..., âgé de 48 ans, entre dans 1e service du professeur Duval, le 25 novembre 1933 , pour une fracture ouverte de la jambe droite. Fracture double des diaphyses tibiale et péronière avec chevauchement en baïonnette des fragments tibiaux ei péroniers. Intervention d'urgence, mise à plat ; on immobilise sur attelle de Boppe avec traction par broche de Kirschner. On enlève la broche le 25 janvier 1934. Le 27 janvier, pose d'un plâtre. Le 5 mars, appareil de Quénu avec étrier de marche ; la suppuration a diminué, mais il y a de l'hydarthrose du genou droit. Le 30 avril, mise à plat de la plaie, ablation de petits séquestres osseux. Le 8 mai, première application de larves de Lucilia sericata. On fait ainsi en tout cinq applications de larves, à raison d'une par semaine ; chaque fois, les larves sont laissées en place pendant quatre jours. Pendant le traitement, on constate d'abord une suppuration très abondante et très liquide, puis le bourgeonnement commence et la plaie se comble peu à peu, sans fistule ; à la fin du traitement, l'état local est excellent, la suppuration tarie. Légère mobilité transversale de la jambe. On n'a constaté aucune poussée de température au cours du traitement. Le 15 juin, le malade quitte l'Hôpital avec un appareil de marche de Delbet.

Obs. VII. Docteur R. Kaufmann. - R. C..., âgé de 34 ans, sans aucun antécédent d'ordre général ni pulmonaire. Ancien sportif qui tend à T'obésité (95 kilos pour $1 \mathrm{~m}$. 61). Le 6 décembre 1933, il se couche ayant « pris froid à la chasse ». Malaise intense et progressif, toux, point de côté ; on pense à une pneumopathie aiguë. En peu de jours, l'état général devient mauvais; température à $39^{\circ}$, expectoration jaune, épaisse, horriblement fétide; on y décèle des streptocoques et des spirilles. M. le $\mathrm{D}^{r}$ Pierre Bourgeois, médecin des Hôpitaux, hospitalise le malade à l'Hòpital Foch, avec le diagnostic de gangrène aiguë du poumon. Le 12 , le 13 et le 14 janvier 1934 , petites hémoptysies; le 15 janvier, hémoptysie que le $\mathrm{D}^{r}$ Bourgeois estime à un litre. Le 23 janvier, une radiographie établit l'étendue des lésions et ne montre pas l'existence de cavité dans le parenchyme. 
Le 3 mars 1934, le malade est consulté par le $\mathrm{D}^{\mathrm{r}}$ Baumgartner qui conseille l'opération. Le 11 mars 1934, opération par le $\mathrm{D}^{\mathrm{r}}$ Kaufmann.

Le 16 mars, dissection au thermocautère d'une rondelle de cortex aussi large que possible et épaisse d'un centimètre; il ne vient ni sang, ni air, ni pus, mais une sérosité œdémateuse putride et l'on voit le parenchyme carnifié parsemé de taches vertes. On place dans la cavité un millier environ de larves de Lucilia sericata. Le surlendemain uns sécrétion considérable a noyé la plupart des larves, mais on peut noter trois faits : $1^{\circ}$ la fétidité a disparu; $2^{\circ}$ la plaie de la paroi thoracique est indolore, sans œdème et rose sous un enduit couenneux, peu adhérent ; $3^{\circ}$ la cavité pulmonaire a pris les dimensions d'un petit poing ; plusieurs fistulettes bronchiques s'y font entendre.

On fait ainsi successivement trois applications d'environ mille larves de Lucilia sericata; chaque fois les larves sont laissées en place pendant quatre jours. Toute la face médiastinale du poumon est nettoyée et l'on voit des larves ramper sur l'oreillette. Le malade, qui va mieux, est rentré chez lui, mais il est évident qu'il persiste un foyer fétide intrapulmonaire supérieur et externe par rapport au foyer précédemment décrit.

Le 21 avril, nouvelle intervention.

Le 23 avril, on place dans la cavité environ deux mille larves de Lucilia sericata; cette fois deux applications sont suffisantes. Quelques larves vivantes sont parfois rejetées par l'expectoration. Le 30 avril, le malade est rentré chez lui. On fait une radiographie. Cinq jours après, il est apyrétique; il ne tousse ni ne crache. Vers le 10 mai, la fistule bronchique était fermée.

Les observations qui suivent se rapportent à des malades soignés à l'aide d'applications locales d'extraits aqueux de larves de Lucilia sericata préparés par nous sous la direction de notre maître, le professeur E. Brumpt, au laboratoire de Parasitologie de la Faculté de médecine de Paris.

OBs. VIII. Service du prof. Mathinev. - P..., àgé de 23 ans. - Plaie superficielle extrémité inférieure de la jambe gauche, 10 centimètres sur 5 centimètres, d'origine tuberculeuse. Plaie atone datant du 10 décembre 1933, abcès froid sous-cutané. Sphacèle abondant. Quatre pansements à l'extrait ont été faits, deux de 48 heures et deux de 3 jours. La suppuration devient liquide, le sphacèle disparaît. On constate la cicatrisation. Un traitement à l'extrait de Lucilia sericata pendant 10 jours a amené la guérison.

Obs. IX. Service du Docteur Louste. - A. D..., àgé de 42 ans, entre à l'Hòpital Saint-Louis le 19 juin 1934. Partie moyenne de la face antérieure interne de la jambe gauche, ulcération large de $10 \mathrm{~cm}$. 5, à bords francs un peu œdématiés en haut et en dehors. Cet ulcère très doulou- 
reux est nettement divisé en deux régions par un rapprochement des bords un peu au-dessus de la partie moyenne ; le segment supérieur est purulent, l'inférieur présente des bourgeons charnus. Tout autour de cet ulcère la peau est rouge et un peu infiltrée. Cet ulcère est apparu pour la première fois il y a quatre ans, à la Charité. Série de Novar. Pas d'antécédents syphilitiques. Varices.

A partir du 22 juin, pansements aux extraits de Lucilia sericata, on fait des applications de 48 heures. Au deuxième pansement, début de bourgeonnement qui néçessite bientôt l'emploi de nitrate d'argent. La peau avoisinante n'est plus infiltrée. Aux premiers pansements, suppuration abondante, puis disparition de l'infection. L'ulcération n'est plus douloureuse. Le 3 juillet, le fond de l'ulcération est remonté au niveau de la peau : le bourgeonnement est intense. Quelques rares points de suppuration sont constatés. On a fait 5 pansements aux extraits. Plaie notablement rétrécie (1 centimètre sur tout le pourtour). La peau avoisinante est parfaitement saine.

Obs. X. Docteur Hartglas. - M. M..., ouvrier agricole, 54 ans, vient nous voir pour la dixième fois pour ses ulcères de jambes; tout traitement paraissait insuffisant. Le début de ces ulcères remonte à 1926 (il y' a 8 ans) à la suite des gelures contractées pendant la guerre. A ce moment, il entre à l'Hòpital de Meaux avec une gangrène des trois derniers orteils de la jambe gauche. En 1928, les deux jambes présentaient des ulcères variqueux, d'abord de minime importance, puis prenant une extension assez considérable. Cette fois-ci, le 26 mai 1934, il entre de nouveau dans notre service, parce que son ulcère de la jambe gauche est infecté et suppure. Notons tout d'abord que les membres inférieurs ne présentent pas ou presque pas de varices. L'ulcère en question est assez étendu, occupant en longueur toute la surface du tiers inférieur de la jambe gauche, longue d'environ 10 centimètres, allant en largeur jusqu'à la face postérieure s'arrêtant en avant à la crête tibiale. Tout autour de la plaie on constate une coloration brun-violacée très pigmentée de la peau, qui est lisse, craquelée, luisante, tendue, collant aux tissus sousjacents. Les bords de cette ulcération sont irréguliers, taillés à pic, très haut et non décollès. Le fond de la plaie n'est pas très profond, mais il présente de nombreux points jaunàtres qui sont des foyers de suppuration. Le pus qui s'étale sur la plaie est fétide, gris-verdâtre, formant même des fausses membranes. L'examen général du sujet nous apprend qu'il s'agit d'un diathésique avéré : atteint d'asthme, emphysème, migraine. L'irrégularité de la plaie, son aspect, sa résistance à la cicatrisation aux moyens médicaux mis en usage, nous ont incités à chercher les tests d'une spécificité chez ce malade. Tous les examens cliniques se sont montrés négatifs à ce propos. De même, les examens de laboratoire dans le sang et dans le liquide céphalo-rachidien ont donné un Wassermann, Kahn, Meinicke négatifs. Aucun spirochète décelable. Le benjoin colloïdal est négatif. La syphilis étant éliminée, on se décide à soigner 
cet ulcère avec de l'extrait de larves de Lucilia sericata. Le 26 mai, première application d'extrait. Le $28 \mathrm{mai}$, deuxième application d'extrait. La plaie a déjà un autre aspect : le pus est comme fluidifié, plus clair. Le 31 mai, troisième application d'extrait. Tout le pus a disparu et l'on aperçoit le fond de l'ulcère nettoyé, rose, bourgeonnant. Le 3 juin, quatrième et dernière application d'extrait. La suppuration est complètement disparue, la plaie est propre, rose, recouverte même d'une fine pellicule épidermique. Les bords de l'ulcère, qui au début étaient saillants, tombant à pic, se fondent; se nivellent et avancent progressivement vers le centre de la plaie. Tout est en voie de guérison, la plaie ne demande qu'à cicatriser. On continue des pansements de propreté non adhésifs avec le Tulle gras Lumière. Pour hâter la cicatrisation et pour mieux nourrir les téguments, on institue également un traitement à l'acecholine.

Obs. XI. Docteur M. Ombrédanne. - Mme D..., âgée de 58 ans, atteinte d'un cancer du maxillaire supérieur. Opérée le 15 mai 1934 par le $\mathrm{D}^{\mathrm{r}}$ Hautant. Le 4 juin, sphacèle sur toute l'étendue de la plaie. Odeur nauséabonde. Pansement avec 15 centimètres cubes d'extrait de larves de Lucilia sericata. Le 5 juin, disparition du sphacèle qui est remplacé par un écoulement purulent. Persistance de l'odeur. Pansement avec 15 centimètres cubes d'extrait. Le 6 juin, écoulement plus liquide, persistance de l'odeur. Pansement avec 20 centimètres cubes d'extrait. Le 7 juin, la cavité est entièrement nettoyée ; il persiste un léger écoulement très liquide. Début de bourgeonnement, disparition de l'odeur. On continue les pansements pendant deux semaines et on obtient une cavité parfaitement propre, à fond bourgeonnant, sans suppuration et sans odeur.

Obs. XII. Docteur M. Ombrédanne. - C..., âgé de 43 ans, entre dans le

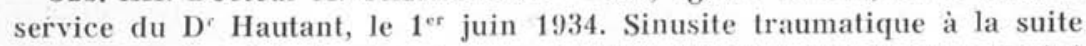
d'un coup de pied de cheval reçu en 1918. Traité médicalement. Opéré a Rennes, en décembre 1919. Suppuration chronique par une fistulisation de la cicatrice au niveau de l'angle interne de l'œil droit. Entre dans le service le $1^{\text {er }}$ juin 1934, opéré le 7 . A la suite de l'intervention, suppuration abondante de la plaie, principalement au niveau de l'ancienne fistule.

Le 16 juin 1934, injection dans le trajet fistuleux de 3 centimètres cubes d'extrait aqueux de larves de Lucilia sericata. Pansement avec compresses imbibées du produit. Le 17 juin, disparition totale de la douleur. Le 18 juin, liquéfaction du pus - mèche dans le trajet - nouveau pansement. La cicatrisation était parfaite le cinquième jour.

Obs. XIII. Docteur M. Ombrédanne. - D..., âgé de 46 ans, entre dans le service du $\mathrm{D}^{\mathrm{r}}$ Hautant, pour sinusite frontale gauche traumatique, à la suite d'un accident survenu le 18 février 1933. Première entrée dans le Service, le 9 mars 1934. Opéré le 10, sorti le 17. Deuxième entrée dans 
le service, le 18 juin. Seconde intervention ; sorti le 25 juin. Troisième entrée dans le service, le 29 juin 1934, pour : douleurs violentes; volumineuse collection suppurée de toute la région orbitaire supérieure, située au niveau de la cicatrice antérieure et empiétant largement sur la paupière.

Le 30 juin, ponction de la tuméfaction au niveau de la cicatrice et de la paupière. On retire ainsi 6 à 7 centimètres cubes de pus. On injecte 5 centimètres cubes d'extrait aqueux de larves de Lucilia sericata. On complète le pansement avec des compresses imbibées de ce produit. Le 31 juin 1934, disparition de la tuméfaction et de la douleur. Persistance d'un peu d'œdème et d'inflammation. Le 2 juillet, nouvelle ponction ne donnant pas de pus. Injection de 3 centimètres cubes d'extrait. Le 3 juillet, la ponction ne donne toujours pas de pus. Injection de 3 centimètres cubes d'extrait. Les douleurs ont presque complètement disparu. Le 4 juillet, il persiste seulement un peu d'inflammation en voie de disparition.

Obs. XIV. Docteur M. Ombrédanne. - L. P..., entre dans le service du $\mathrm{D}^{\mathrm{r}}$ Hautant pour ostéite traumatique, le 13 juin 1934. Opéré le 15 juin par le $\mathrm{D}^{r}$ Marcel Ombrédanne. Enfoncement du frontal en février 1934. Atrophie optique avec cécité gauche complète. Ostéite chronique. Large perte de substance de la racine du nez intéressant la peau, les plans sous-jacents, la paroi antérieure des deux sinus frontaux et les deux ethmoïdes antérieurs.

Le 19 juin, pansement avec extrait de larves de Lucilia sericata. Le 21 juin, deuxième pansement; le pus devient très liquide. Le 23 juin, troisième pansement; absence totale de pus. Début de bourgeonnement sur les lèvres externes de la plaie. Le 24 juin, quatrième pansement : début de cicatrisation sur toute l'étendue de la plaie.

Obs. XV. Docteur M. Ombrédanne. - Mme B..., âgée de 60 ans. Tumeur de la parotide. Opérée le 27 avril par le $\mathrm{D}^{r}$ Ombrédanne. Plaie sphacélée, non fétide.

Du 19 au 24 mai 1934, pansements avec extrait de Lucilia sericata. Disparition du sphacèle, suppuration abondante très liquide.

Obs. XVI. Docteur M. Ombrédanne. - W. R..., àgée de 4 ans $1 / 2$. Mastoïdite bilatérale. Ecoulement de l'oreille droite. Le 27 décembre 1933, entre à l'Hôpital Trousseau. Trépanation mastoïde droite. Scarlatine, diphtérie. Le 15 février 1934, entre à l'Hôpital Tenon. Ecoulement et douleurs à gauche. Trépanation mastoïde gauche. Le 12 mars 1934, sort de l'hôpital, l'écoulement d'oreille persiste, plaies purulentes. Le 20 avril, nouvelle intervention. Persistance de l'écoulement de l'oreille droite, plaies purulentes.

Le 19 mai, pansement avec mèches d'oreille imbibées d'extrait de Lucilia sericata dans le conduit. Compresses également imbibées sur chaque plaie. Le 20 mai, l'écoulement est plus liquide, on constate 
l'absence de pus sur les plaies. On fait un nouveau pansement selon la même technique. Le 21 mai, l'écoulement est devenu très liquide et inodore. Les plaies commencent à bourgeonner. Le 22 mai, nouveau pansement. Les plaies sont parfaitement cicatrisées, l'écoulement persiste mais inodore. Le 29 mai l'écoulement a diminué. On ne continue pas le traitement faute d'extrait. Reprise du traitement trois semaines plus tard, le 16 juin 1934. A cette date l'écoulement était redevenu épais et fétide. Le 19 juin, diminution de l'écoulement, liquéfaction, disparition complète de la fétidité.

Obs. XVII. Docteur M. Ombrédanne. - Mme C..., atteinte de tumeur maligne du nez, opérée par le $\mathrm{D}^{r} \mathrm{M}$. Ombrédanne, le 21 septembre. Résection au bistouri électrique, point suspect de la partie antérieure de la cloison dans l'angle antérieur à la face profonde du lobule ; coagulation prudente. Jusqu'au 13 octobre pansements divers: tulle gras, puis Dakin. Cicatrisation peu rapide, persistance des croûtes.

Le 13 octobre, il y a un volumineux magma purulent de croûtes à demi desséchées. Sans y toucher, pansement avec deux ampoules d'extrait. Le 15 octobre, quand on enlève le pansement, on constate sur les mèches la présence d'un pus semi-liquide, non fétide, la plaie se présente parfaitement propre, la muqueuse apparaît d'un joli rouge vif. On continue tous les deux jours les pansements à l'extrait. Dernier pansement, le 5 novembre : les bourgeons sont roses et serrés, la plaie parfaitement propre, début d'épidermisation.

Il ne nous reste plus, pour terminer cette étude clinique, qu'à étudier une observation obtenue en médecine vétérinaire; nous verrons alors que les résultats obtenus sont, dans leurs grandes lignes et dans la marche de la guérison, absolument identiques à ceux que nous venons de publier ; on peut constater sur l'homme et sur les animaux le parallélisme de l'action des larves de Lucilia sericata, qu'il s'agisse des larves elles-mêmes ou de leurs extraits aqueux.

OBs. XVIII. Capitaine Vétérinaire Vectes. - Clos Vougeot, cheval hongre, âgé de 10 ans, du $24^{\circ}$ Régiment d'Infanterie. L'animal a été blessé le 2 juillet 1934, après-midi, dans une collision avec une voiture automobile. Le membre antérieur gauche, seul lésé, présente trois blessures: l'une, la plus importante, au niveau de la partie antérieure et médiane de l'avant-bras, se caractérise par une section des téguments en forme d'Y très évasé et mesurant 30 centimètres pour les branches transversales et 15 centimètres pour la branche verticale. Les deux autres blessures, situées l'une au niveau du tiers inférieur du tendon, l'autre à la face externe du boulet, mesurent 7 centimètres de long. Elles sont disposées verticalement et se présentent, comme la première, sous 
forme de section du tégument. L'animal est conduit à l'infirmerie vers dix heures du soir. Bien que les lèvres des plaies soient déjà rétractées, on procède à une suture à épingles entortillées, après nettoyage chirurgical. Dans la nuit du 4 au 5 juillet, malgré le collier à chapelet qui lui avait été mis, le cheval arrache les sutures à coups de dents. Le 5 au matin, la plaie de l'avant-bras est largement ouverte, sanguinolente, ses lèvres sont déchiquetées et les parties musculaires sous-jacentes sont entamées. Les plaies de la partie inférieure du membre sont également arrachées. Dans les jours qui suivent, les lésions sont le siège d'une suppuration abondante. Le pus est sanieux et malodorant. Tout le membre est fortement œdématié ; l'état général devient mauvais sans pourtant que la température dépasse $38^{\circ}$. Le membre présente alors: une plaie antérieure et supérieure de 15 centimètres de haut sur 32 centimètres de large. Deux plaies postérieures et inférieures de 7 à 8 centi. mètres de haut sur 3 à 4 centimètres de large.

La première application de larves vivantes a lieu le 12 juillet sur la plaie antérieure. Les deux plaies inférieures sont pansées à l'aide d'une compresse imbibée d'extraits aqueux de larves. Le pansement consiste dans un rembourrage cylindrique allant du sabot jusqu'à quelques centimètres du bord inférieur de la plaie de l'avant-bras. Le griliage de fil de bronze destiné à empêcher les souillures extérieures tout en laissant les larves à l'air, est cousu en manchon sur un bourrelet de coton cardé entouré de gaze, et qui est lui-même assujetti à quelques centimètres au-dessus du bord supérieur de la plaie. Le bord inférieur est maintenu sur le pansement du bas par un tour de bande. La rigidité du grillage ne paraissant pas suffisante pour maintenir l'appareil en place, deux bandes de ruban de fil sont disposées en bretelles croisées au-dessus du garrot et cousues sur le bourrelet. Pour éviter que les mouvements du cheval n'entrainent la destruction du pansement qui doit rester en place plusieurs jours, on fixe par un bandage, à la partie postérieure du membre, une tige de bois destinée à empêcher la flexion du genou. Le pansement est enlevé le 16 juillet : le tampon de coton hydrophile qui avait été placé au-dessous de la plaie de l'avant-bras pour collecter les sécrétions est gorgé de pus liquide de bonne nature; aucune larve n'est retrouvée sur la plaie ni dans le pansement. Après détersion à l'eau bouillie, on constate que la plaie est fortement améliorée : elle est couverte uniformément de petits bourgeons roses et serrés, sans aucune exubérance. Ces bourgeons offrent cette particularité de n'être pas fragiles, comme cela se produit d'habitude; ils n'ont aucune tendance à saigner au moment du lavage et du nettoyage de la plaie. Les plaies du bas sont plates et couvertes d'un pus léger qui semble plutòt une sécrétion cellulaire que du pus proprement dit. L'état général s'est considérablement amélioré; l'appétit est revenu, l'œdème du membre s'est presqu'entièrement résorbé, l'odeur fétide a entièrement disparu.

Pansement sec sur la plaie supérieure ; pansements à l'extrait de lar-ves sur les plaies inférieures. 19 juillet : Un sillon d'épidermisation s'est 
dessiné sur le pourtour des trois plaies; celles-ci ont déjà notablement diminué de surface. Etat général excellent. On procède alors à une deuxième application de larves vivantes de Lucilia sericata sur la plaie supérieure ; on continue le pansement des plaies inférieures à l'extrait. 23 juillet : On enlève les larves. Les trois plaies continuent à s'améliorer simultanément. 26 juillet: Vu l'excellent état, général et local, de l'animal, on abandonne les larves vivantes, et les trois plaies sont soignées par des pansements à l'extrait de larves. 10 août : Les plaies inférieures sont entièrement cicatrisées. La plaie supérieure est pansée à l'extrait. 14 août: Dimensions de la plaie supérieure : 17 centimètres sur 9 centimètres. Pansement à l'extrait. 22 août : Pansement à l'oxyde de zinc qui est continué jusqu'au 4 septembre ; à cette date la plaie est laissée à i'air, recouverte d'une préparation au Bleu de Méthylène, Glycérine et Borate de Soude; elle mesure alors 15 centimètres sur 6 centimètres. 15 septembre: La plaie ne mesure plus que 10 centimètres de long et 3 centimètres dans sa plus grande hauteur. Elle marque une tendance caractéristique à devenir linéaire.

\section{Conclusions}

Le traitement par les larves vivantes de Lucilia sericata et par leurs extraits aqueux a déterminé, immédiatement après son application, une amélioration extrêmement nette, alors que les traitements médicamenteux étaient restés sans effet. L'amélioration locale s'est accompagnée d'une amélioration rapide de l'état général du sujet.

Le bourgeonnement est très régulier ; les bourgeons sont petits, extrêmement denses et résistants, n'ayant pas, comme les bourgeons ordinaires, tendance à saigner au moinde contact ; c'est vraisemblablement à cette nature des bourgeons qu'il faut attribuer la régularité de la cicatrisation.

Il semble que ce traitement, qu'il s'agisse de l'application des larves vivantes ou de leurs extraits, détermine la formation de cicatrices linéaires dont l'intérêt esthétique mérite, même en médecine vétérinaire, d'être retenu.

\section{BIBLIOGRAPHIE}

BAEn (W. S.). - Abstract of his discussion of paper on osteomyelitis. South Med. Journal, XXII, 1921, p. 582.

The treatment and cure of the disease known as osteomyelitis. Hearings before the committee on World War Veteran's legislation, House of representatives, seventy-first Congress, Second session, april 17, 1930. U. S., Govern. printing office. 
Baer (W. S.). - Proc. Interst. Post-Grad. M. Ass., N. America, 1930, p. 371.

- The treatment of chronic osteomyelitis with the maggot (larva of the blow fly). Journal Bone and Joint Surg., XIII, 1931, p. 438.

Bishopp (F. C.). - Flies which cause myasis in man and animals. Some aspects of the problem. Journal Econ. Ent., VIII, n' ${ }^{\circ} 3,1915$, p. 317-329.

Brumpt (E.). - Notes et observations sur les maladies parasitaires. V. Fréquence de la myiase en Abyssinie. Arch. de Parasit., IV, 1901, p. 563.

- Précis de Parasitologie, $1^{\text {re }}$ édition, Masson édit. Paris, 1910, p. 614, 615.

- Les myiases chirurgicales. Bull. Acad. Méd., CIX, 1933, p. 891-899.

- Utilisation des larves de certaines mouches pour le traitement de l'ostéomyélite et de diverses affections chirurgicales chroniques. Ann. Parasit. Hum, el comp., XI, 1933, p. 403-420.

Buchman (J.) et Blair (J. E.). - Maggots and their use in chronic osteomyelitis. Surg. Gynec. and Obst., IV, août 1932, p. 177-190.

Durousseau-Dugontier (R.). - Technique de préparation et utilisation en chirurgie des larves, de Lucilia sericata. M. Vigné, édit., Paris, 1934.

Goldstern (H. I.). - Maggots in treatment of infected wounds, complicated fractures, osteomyelitis and tuberculous abscesses. Ann. Surg., XCIII, avril 1931, p. 953-955.

- Maggot́s. J. Amer. Med. Assoc., XCIV, 1931, p. 290.

- Maggots in the treatment of infected wounds. Med. Rev. of Rev., XXXVIII, juin et juillet 1931 , p. 327 et 361 .

- Maggots in treatment of wounds and bone infections (historical). J. Bone and Joint. Surg., XIII, juillet 1931, p. 476-478, et aussi Med. J. and Rec., CXXXIV, oret. 1931, p. 329-330.

Hopkins (F. M.). - Use of maggots in surgical therapy. J. Nat. M. A., XXIV, nov. 1932 , p. $15-17$.

LARREY (Baron D. J.). - Relation historique et ehirurgicale de l'expédition de l'armée d'Orient en Egypte et en Syrie, Paris, 1803, p. 116.

Lenormant (Ch.). - Ostéomyélite chronique du fémur traitée par les larves de mouches. Bull. et Mém. de la Soc. Nat. de Chirurgie, LX, ${ }^{\circ} 1$, 20 janvier 1934, p. 52 .

Mathieu (P.). - Discussion à propos de la communication précédente.

MONoD (R.). - Idem.

Livingston (S. K.). - Maggots in treatment of chronic osteomyelitis, infected wounds and compound fractures. Analysis based on treatment of 100 cases with preliminary report on isolation and use of active principle. Surg. Gynec, and Obst., LIV, avril 1932, p. 702-706. - Med. Bull. Vet. administ., IX, july 1932 , p. 1-6.

- Maggot treatment of osteomyelitis. J. Amer. Med. Assoc., Correspondance, XCVIII, 1932, p. 1585.

\section{EXPLICATION DE LA PLANCHE I}

Photographies en séries faites au cours du traitement de la malade de lobservation 5: 1, 29 novembre $1933 ; 2,4$ décembre $1933 ; 3,5$ février 1934 ; 4, 23 avril 1934. 

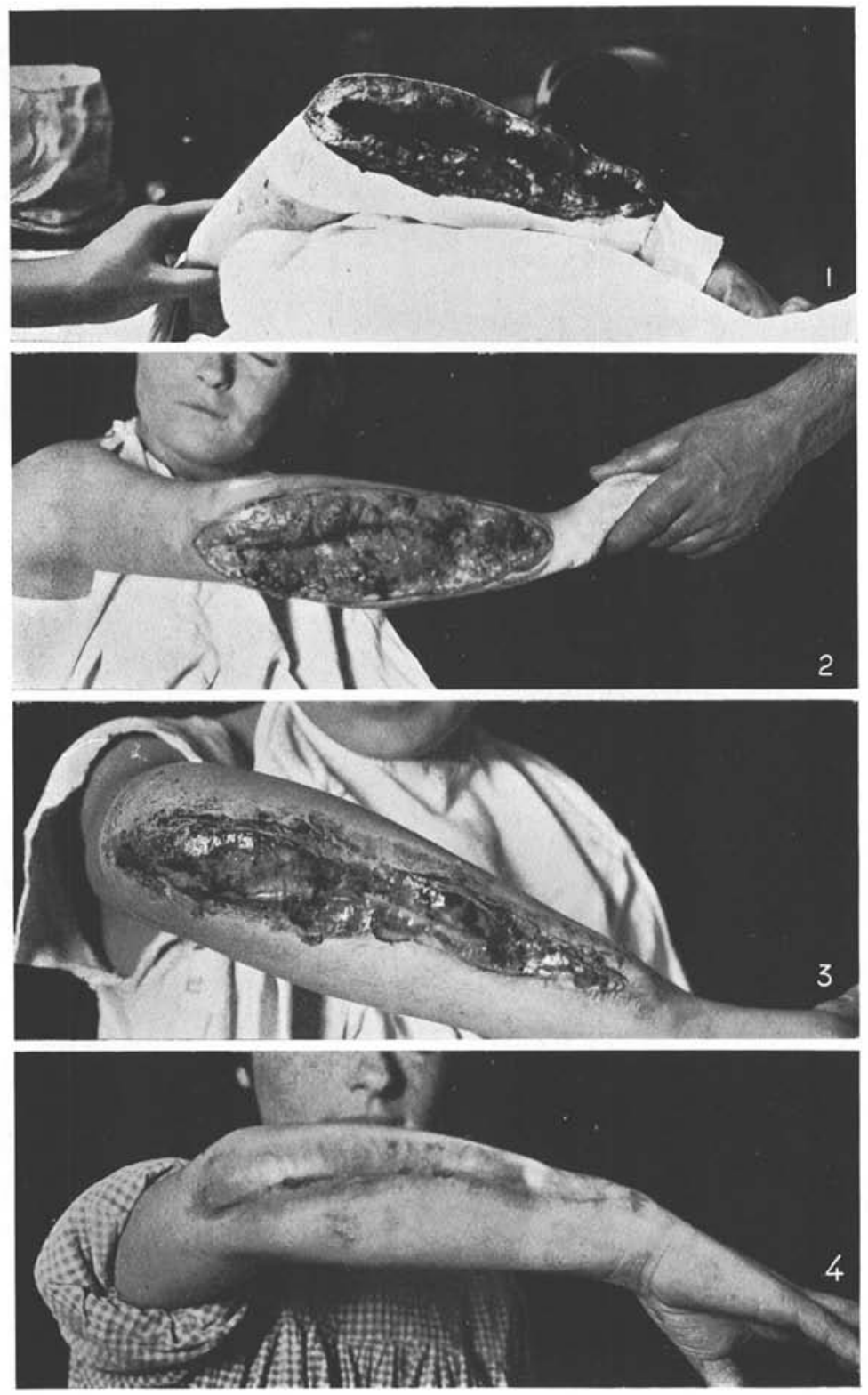

Masson et Cie, Editeurs 
Livingston (S. K.) and Prince (L. H.). - Treatment of chronic osteomyelitis with special reference to the use of the maggot active principle. $J$. Amer. Med. Assoc., XCVIII, avril 1932', p. 1143-1149.

Ombrédanne (L.). - A propos du traitement de l'ostéomyélite chronique par les larves de Lucilia sericata. Bull. et Mém. de la Soc. Nat. de chirurgie, LX, $\mathrm{n}^{\circ} 4,10$ février 1934 , p. 210.

Ombrédanne (M.), Metrey (G.) et Maurice (A). - Malades traités par un extrait de larves de Lucilia sericata. Présentation de malades à la Société d'oto-rhino-laryngologie de Paris. Sćance du 9 juillet 193/.

PARÉ (Ambroise). - Les cuvres d'Ambroise Paré, 2" édit., Gabriel Buon, édil., Paris, 1579, p. 366.

PARÉ (Ambroise). - (Traduction anglaise). The workes of that famous chirurgion Ambroise Parey, translated out of latine and compared wilh the French by Th. Johnson. Londres, 1634, p. 372.

Parton (W. S.) et Evans (A. M.). - Insects, ticks, mites and venomous animals. Grubb édit., Croydon, 1929, p. 459.

Robinson (W.). - The use blow fly maggots in the treatment of osteomyelitis and certain other diseases. Bureau of Enl., Washington, circ. E-285, $1^{\text {er }}$ mars 1932.

The rearing of blow flies and the culture of sterile maggots for use in osteomyelitis. Bureau of Ent., Washington, eirc. E-296, 9 mars 1932.

Vecten et Cosson. - Essai de traitement des plaies du cheval par les larves et les extraits de larves de Lucilia sericata. Présentation à l'Acad. Vétér. de Paris par le vétérinaire général Vivien. Séance dù ' octobre 193\%.

Laboratoire de Parasitologie de la Faculté de médecine de Paris

(Directeur: Prof. E. Brumpt). 